\title{
Las Universidades Populares en Uruguay (1930-1942)*
}

\section{GABRIEL SCAgLIOLA}

Afiliado institucionalmente a los Institutos Normales de Montevideo e Instituto de Formación Docente de Florida (Uruguay). Correo electrónico: gabriel.scagliola@gmail.com. El autor es maestro por los Institutos Normales de Montevideo (Uruguay); magíster en Memoria y Crítica de la Educación por la Universidad de Alcalá de Henares y la Universidad Nacional de Educación a Distancia (Madrid, España); candidato a doctor por la Universidad Nacional de Educación a Distancia (Madrid, España). Entre sus publicaciones recientes tenemos: "Una experiencia desconocida: las Misiones Socio-Pedagógicas en Uruguay (1945-1959)", Revista del IICE No. 44 (2018) y en coautoría "Museo Pedagógico José Pedro Varela: expressando uma comunidade de aspirações!”, Museologia \& Interdisciplinaridade Vol. 8 No. 16 (2019). Entre sus temas de interés se encuentran Educación Popular, Universidades Populares, Misiones Pedagógicas, Cultura material.

Recibido: 3 de febrero de 2019

Aprobado: 19 de marzo de 2019

Modificado: 14 de junio de 2019

Artículo de investigación científica

DOI: https://doi.org/10.15648/hc.38.2021.2812

Este artículo forma parte del proyecto "La Educación Popular en Montevideo: De las Universidades Populares a las Misiones Sociopedagógicas (1930-1959)", financiación propia.

Esta publicación está bajo una licencia Creative Commons Reconocimiento-NoComercial 4.0 (cc) BY-NC 


\title{
Las Universidades Populares en Uruguay (1930-1942)
}

\section{Resumen}

Las Universidades Populares surgen en Francia a finales del siglo XIX y principios del XX, esparciéndose por Europa y Latinoamérica. En Uruguay, en el marco de la lucha por la Reforma Universitaria, estudiantes universitarios, a los que se irán sumando profesionales, intelectuales y trabajadores, organizan en 1930 la primera Universidad Popular, a la que seguirán otras instituciones de este tipo en los años siguientes. El artículo analiza el proceso de creación, expansión y disolución de las Universidades Populares uruguayas. Durante décadas estas Universidades Populares fueron olvidadas; sin embargo, el registro de esas iniciativas existe disperso en archivos particulares y publicaciones diversas. Este trabajo es uno más de esos esfuerzos que se están llevando a cabo para recuperar su memoria.

Palabras clave: Universidad Popular, movimiento estudiantil, extensión universitaria; reforma universitaria, Uruguay, educación de adultos.

\section{People's Universities in Uruguay (1930-1942)}

\begin{abstract}
People's Universities emerged in France in the late nineteenth and early twentieth centuries, spreading throughout Europe and Latin America. In Uruguay, within the struggle for University Reform, university students, joined by professionals, intellectuals and workers, organized in 1930 the first People's University, which would be followed by other institutions of this type in the following years. This article analyzes the creation, expansion and dissolution process of Uruguayan People's Universities. For decades these People's Universities were forgotten; however, register of these initiatives is scattered in private archives and various publications. This work responds to an effort with the intention of recovering their record of events.
\end{abstract}

Key words: People's University, student movement, university extension, university reform, Uruguay, adult education.

\section{As Universidades Populares no Uruguai (1930-1942)}

\section{Resumo}

As Universidades Populares surgem na França no final do século XIX e início do 
XX, expandindo-se pela Europa e América Latina. No Uruguai, no âmbito da luta pela Reforma Universitária, estudantes universitários, aos quais se somarão profissionais, intelectuais e trabalhadores, organizam em 1930 a primeira Universidade Popular. Instituição que seguirão outras Universidades deste tipo nos anos seguintes. O artigo analisa o processo de criação, expansão e dissolução das Universidades Populares uruguaias. Durante décadas estas Universidades Populares foram esquecidas. No entanto, o registro dessas iniciativas está disperso em diversos arquivos e publicações. Este trabalho é um desses esforços que estão sendo feitos para recuperar sua memória.

Palavras-chave: Universidade Popular, movimento estudantil, extensão universitária, reforma universitária, Uruguai, educação de adultos.

\section{Les Universités Populaires en Uruguay (1930-1942)}

\section{Résumé}

Les Universités Populaires naissent en France fin du XIX siècle début du XX siècle, en se répandant en Europe et Amérique Latine. En Uruguay, dans le cadre de la lutte pour la Réforme Universitaire, des étudiants, auxquels se joindront des professionnels, intellectuels et des ouvriers, organisent la première Université Populaire en 1930, laquelle sera suivie par d'autres institutions du même type dans les années successives. L'article fait l'analyse du processus de création, expansion et dissolution des Universités Populaires uruguayennes. Pendant des décennies, ces Universités Populaires ont été oubliées ; pourtant, le registre de ces initiatives est dispersé dans des archives particuliers et des publications diverses. Ce travail est un autre sorti des efforts qui se réalisent pour récupérer leur mémoire.

Mots clés: Université Populaire, mouvement étudiant, expansion universitaire, réforme universitaire, Uruguay, éducation d'adul

\section{INTRODUCCIÓN}

A finales del siglo XIX surgieron las Universidades Populares en Francia, extendiéndose rápidamente por Europa. El proyecto estuvo vinculado al movimiento cultural generado en torno a George Deherme (18761937), obrero autodidacta y propagandista vinculado al pensamiento libertario. La primera Universidad Popular francesa se establece en París el 9 de octubre de 1899, en el No. 19 de la calle Paul Bert. El 
éxito dio origen a la multiplicación y la diversificación de la iniciativa, organizándose entre los años 1899 y 1914 al menos 230 Universidades Populares en Francia. Leopoldo Palacios Morini (1876-1952), uno de los impulsores de las reformas educativas y sociales del primer tercio del siglo XX en Oviedo (España), agrupa a las Universidades Populares de Francia en tres categorías: las Universidades Populares de La Coopération des Idées, destinadas a las clases populares independientemente de su ideología; las llamadas Universidades Populares de "partido", de carácter laico, dirigidas a grupos de obreros pertenecientes a determinados grupos políticos o asociaciones al servicio de la lucha de clases; y los institutos populares, "enseñanza mutua de compenetración y amistad: ni confesional ni neutra (hecha por católicos, según métodos racionales); acción social"”.

En Latinoamérica, durante la primera mitad del siglo XX comenzaron a tomar forma desde el movimiento estudiantil las primeras propuestas de vinculación de los institutos de educación superior con la sociedad. El eje articulador de estos planteos fueron los congresos estudiantiles, que permitieron el debate de ideas tales como la extensión universitaria, las Universidades Populares, la difusión y el acceso a la cultura.

El Primer Congreso Internacional de Estudiantes Americanos, reunido en Montevideo entre el 26 de enero y el 2 de febrero de 1908, propuso la instrumentación de programas dirigidos a "difundir la cultura intelectual en la sociedad"2. Dos años después, en Buenos Aires, el Segundo Congreso Internacional de Estudiantes Americanos resolvía recomendar la extensión universitaria como el medio para obtener "la elevación intelectual y moral del pueblo”, y establecía que en los lugares donde no hubiere universidades, los estudiantes debían ayudar a la fundación "de salones ó clubs obreros” y realizar "cátedras populares y conferencias públicas"3. A partir del Primer Congreso Nacional de Estudiantes Universitarios, organizado por la Federación Universitaria

1 Leopoldo Palacios Morini, Las Universidades Populares (Valencia: F. Sempere y Compañía Editores, 1908), 195.

2 "Relación Oficial del Primer Congreso Internacional de Estudiantes Americanos", Evolución (marzo-junio de 1908): 33.

3 "Conclusiones aprobadas en las sesiones plenas del II Congreso Internacional de Estudiantes Americanos reunido en Buenos Aires en 1910”, Evolución (enero, 1912): 88. 
Argentina en 1918, la extensión universitaria estará en "programas de los centros y federaciones estudiantiles de toda nuestra América"4. En 1921, el Primer Congreso Internacional de Estudiantes reunido en México, manifestaba: "Es una obligación de los estudiantes el establecimiento de universidades populares, que estén libres de todo espíritu dogmático y partidista y que intervengan en los conflictos obreros inspirando su acción en los modernos postulados de justicia social" 5 .

Entre 1918 y 1925 las Universidades Populares organizadas por el movimiento estudiantil surgieron por toda Latinoamérica: en 1918 se fundó la Universidad Popular Lastarria por iniciativa de la Federación de Estudiantes de la Universidad de Chile; el Primer Congreso Nacional de Estudiantes Peruanos realizado en Cuzco, entre el 11 y el 20 de marzo de 1920, aprobaba la creación de las Universidades Populares; la Universidad Popular de Guatemala funcionaba con el apoyo de Miguel Ángel Asturias desde 1923; en Cuba, a iniciativa de Julio Antonio Mella, se fundaba la Universidad Popular "José Martí", el 3 de noviembre de $1923^{6}$.

\section{URuguay: La ÉPOCA batllista (1903-1933)}

José Batlle y Ordóñez accedió a la presidencia de Uruguay en dos oportunidades: en 1903 y en 1911. Hasta su fallecimiento en 1929 fue una figura destacada, hasta el punto de que se suele denominar al período que abarca las tres primeras décadas del siglo XX con el nombre de época batllista ${ }^{7}$. Durante esos años Uruguay experimentó una serie de reformas que abarcaron todos los planos. A nivel político, el voto universal masculino y secreto democratizó la participación política de la ciudadanía, a la vez que restringió el fraude electoral. En el plano económico, el batllismo impulsó la industria nacional mediante el

4 Gabriel del Mazo, Estudiantes y gobierno universitario, 1946. Citado por Jorge Bralich, La Extensión Universitaria en el Uruguay (Montevideo: Comisión Sectorial de Extensión y Actividades en el Medio, 2007), 22.

5 Gabriel del Mazo (comp.), La Reforma Universitaria (La Plata: Centro de Estudiantes de Ingeniería, 1941), Tomo II, 86-88.

6 Gabriel del Mazo (comp.), La Reforma Universitaria, Tomo II.

7 Véase Benjamín Nahum, La época batllista, 1905-1929, Tomo 8 (Montevideo: Ediciones de la Banda Oriental, 2011). 
proteccionismo y el fomento de la producción interna, enfrentándose a los intereses de los capitales extranjeros. La política de estatalización de los servicios esenciales efectuada por el gobierno persiguió un doble objetivo: brindar un mejor servicio a la población (telégrafos, electricidad, ferrocarriles, etc.), mejorando la calidad y bajando los costos; e impedir que las ganancias de estas empresas emigraran a su país de origen, pudiendo reinvertirlas en Uruguay ${ }^{8}$. Las leyes sociales apuntaron a otorgar garantías y mejores condiciones de trabajo a los obreros (ejemplo: ley de ocho horas) y una superior calidad de vida al culminar la etapa laboral (ejemplo: Caja de Jubilaciones) ${ }^{9}$.

En lo educativo, la Ley Orgánica de 1908 creaba los consejos de Facultades por debajo del Consejo Universitario ${ }^{10}$, y también incluía la representación estudiantil indirecta ${ }^{11}$. Las Facultades aumentaron: en 1915 la de Matemáticas se dividió en Arquitectura e Ingeniería; en 1925 se constituyó la de Agronomía sobre la base de la Escuela; en 1929 se crearon las Facultades de Odontología y de Química y Farmacia, al desprenderse de la de Medicina; en 1932, sobre la base de la Escuela de Comercio, se creó la Facultad de Ciencias Económicas y de Administración; en 1933 la de Veterinaria surgió de la Escuela ${ }^{12}$.

Durante la época batllista se elaboraron diversos proyectos orientados a expandir la enseñanza secundaria. El 4 de mayo de 1911 el Poder Ejecutivo remitió un mensaje a la Asamblea General donde proponía la creación de un establecimiento de educación media en cada una de

8 Véase Jaime Yaffé, Ideas, programa y política económica del batllismo. Uruguay, 1911-1930 (Montevideo: Instituto de Economía, Facultad de Ciencias Económicas y de Administración, 2000). También Benjamín Nahum, La época batllista, 92-106 y 123-139.

9 Véase Carlos Rama, Batlle: la conciencia social. Enciclopedia Uruguaya, Vol. 34 (Montevideo: Arca, 1968), 73-76.

10 La Ley Orgánica aprobada en 1908 daba autonomía técnica al gobierno de las Facultades: "El rector y el Consejo Central -que se integraría con los decanos y con delegados de Facultades- conservaron diversas atribuciones más, descargándose de todos modos en las Facultades las funciones más importantes”. Juan Oddone y Blanca París de Oddone, Historia de la Universidad de la República. Tomo II: La Universidad del militarismo a la crisis (Montevideo: Ediciones Universitarias, 2010): 81.

11 Los estudiantes designaban a una persona "que esté científicamente a la altura de los demás miembros de la Facultad" para que los representara en el Consejo. Diario de Sesiones de la Cámara de Representantes, 9 de mayo de 1908, 87. Citado en Vania Markarián, María Eugenia Jung e Isabel Wschebor, 1908. El año augural (Montevideo: Universidad de la República, 2008), 25.

12 Arturo Ardao, La Universidad de Montevideo. Su evolución histórica (Montevideo: Ediciones Universitarias, 2008), 13-14. 
las capitales departamentales ${ }^{13}$. La intención era evitar el desarraigo de los adolescentes de su medio, y facilitar "el acceso de un mayor número de jóvenes a la enseñanza, al no imponer de hecho la alternativa del traslado a Montevideo o la asistencia al instituto privado". El debate del proyecto fue breve y se sancionó el 30 de diciembre, promulgándose el 5 de enero de 1912. El 8 de mayo de 1912 se aprobaba la ley que creaba la Sección Enseñanza Secundaria y Preparatoria destinada al sexo femenino en la Universidad de la República ${ }^{14}$. El Mensaje del Poder Ejecutivo expresaba: "Se quiere dar a la mujer elementos de que hoy - con notoria injusticia- se ve privada, a fin de que pueda actuar en las mismas profesiones que hasta ahora solo en casos excepcionales puede ejercer"'15. En 1914 el Poder Ejecutivo presentó al Parlamento el proyecto para exonerar de tributos a los estudiantes de la enseñanza secundaria, preparatoria y con la posibilidad de ir otorgando la gratuidad a los universitarios ${ }^{16}$. La oposición de algunos legisladores del Partido Nacional al proyecto retrasó la aprobación hasta enero de $1916^{17}$.

La propuesta más importante del período y que ocasionó “más entusiasmo entre los estudiantes" fue la creación de las llamadas "cátedras

13 La República Oriental del Uruguay se encuentra dividida políticamente en dieciocho departamentos. El país contaba entonces con poco más de 1.000 .000 de habitantes y la tasa de analfabetismo registrada en el Censo de 1908 era del orden del 36 \%. Benjamín Nahum (coord.), Estadísticas históricas del Uruguay, 1900-1950. Tomo I: Población y Sociedad, Política, Educación, Estado (Montevideo: Universidad de la República, 2007), 43.

14 "Ley creando una Sección de Enseñanza Secundaria y Preparatoria Femenina" (Montevideo, 17 de mayo de 1912), Anales de la Universidad, Año XVII, Tomo XXII (1912): 345-348. Citado en Luis Ma Delio Machado y Alfredo Alpini, "La educación secundaria y superior entre 1903 y 1935", en Historia de la educación uruguaya. Tomo 3: La educación uruguaya 1886-1930, ed. Agapo Palomeque (Montevideo: Ediciones de la Plaza, 2012), 306 - 309.

15 Diario de Sesiones de la Honorable Cámara de Representantes, 47 ${ }^{a}$ Sesión Extraordinaria, 25/11/1911. Sesiones extraordinarias del primer período de la XXIV Legislatura, Tomo CCXIII, octubre 19 a diciembre 9 de 1911 (Montevideo: El Siglo Ilustrado, 1912), 177.

16 B. Nahum, A. Cocchi, A. Frega y Y. Trochon, Crisis política y recuperación económica, 1930-1958. Historia Uruguaya, Tomo 9 (Montevideo: Ediciones de la Banda Oriental, 2011), 54; Luis Ma Delio Machado y Alfredo Alpini, La educación secundaria y superior entre 1903 y 1935, 301-306. Los estudiantes apoyaron fervientemente la propuesta del Poder Ejecutivo: "El 22 tuvo lugar la manifestación con que los estudiantes de todas las facultades exteriorizaron su simpatía por el último y feliz proyecto del Ministro de Instrucción Pública, doctor Baltasar Brum. A más de mil ascendió el número de los manifestantes, los que partieron de la Plaza Libertad y se dirigieron por la avenida 18 de Julio hasta la plaza Constitución, deteniéndose frente al Club Uruguay, en cuyos balcones se hallaban las estudiantes de la Universidad de Mujeres y varias señoritas que cursan en las Facultades superiores". "La manifestación pro-exoneración de matrículas", Evolución (febrero de 1915): 117.

17 Al igual que el Partido Colorado, es considerado uno de los partidos tradicionales del país. 
libres" en las Secciones de Enseñanza Secundaria y Preparatoria, y en las Facultades de la Universidad de Montevideo. Esta propuesta fue elaborada por Baltasar Brum, mientras desempeñó el cargo de Ministro de Relaciones Exteriores e Instrucción Pública durante la segunda presidencia de José Batlle y Ordoñez (1911-1915) ${ }^{18}$. La Federación de Estudiantes del Uruguay, a través de la revista Evolución, respaldaba el proyecto, ya que suprimía "ese absurdo consagrado en la legislación universitaria, según el cual el catedrático es ‘dueño’ de su cátedra en el sentido más material y más absoluto de la palabra"19. En el articulado del proyecto se establecía que los catedráticos que habían obtenido sus cátedras por concurso continuarían desempeñándolas por diez años, y los nombrados de manera directa, por cuatro años. Finalizado ese plazo, podían seguir renovándola cada cuatro años ${ }^{20}$. Asimismo, se establecían tres tipos de cátedras: a) de materias programadas, en las que se dictarían cursos de acuerdo con el programa del titular; b) de materias que no figuran en el plan de estudios pero que tienen relación con las que se siguen en cada Facultad; y, c) de especialización, para aquellos graduados, o estudiantes, que quieran profundizar los conocimientos en una materia determinada.

\section{Creación y definiciones del Centro de Estudiantes Ariel}

En 1917 se produjeron dos acontecimientos de importancia para el movimiento estudiantil. Por una parte, la disolución de la Federación de Estudiantes del Uruguay (F.E.U.) ${ }^{21}$, que había sido creada en 1909. Su desaparición condujo a la desunión y al aislamiento de los centros

18 Evolución (marzo de 1915): 121-140. La revista Anales de la Liga de Estudiantes Americanos titulaba "Proyecto del Excmo. Señor Ministro de Relaciones Exteriores e Instrucción Pública, doctor Baltasar Brum", Anales de la Liga de Estudiantes Americanos (febrero de 1915): 2.

19 En 1919, El Estudiante Libre, publicación oficial del Centro de Estudiantes de Medicina, va a destacar la importancia de contar con cátedras libres frente a las vitalicias: "La cátedra libre previene a los estudiantes de uno de los grandes males de la cátedra oficial vitalicia, pues los defiende y lo salva en los casos de decadencia y vejez del profesor de la última palabra". "Alegato de la delegación estudiantil", El Estudiante Libre (noviembre 15 de 1919): 5.

20 "Reorganización Universitaria", Evolución (enero de 1915): 4.

21 Fundada en 1909, fue conocida como "La FEU de La Cumparsita", debido a que el famoso tango de Mattos Rodríguez fue compuesto en el local de la Asociación de Estudiantes de Medicina. Entre 1915 y 1917 "La FEU de La Cumparsita" se fue desbaratando por la "indiferencia" de los estudiantes y la "falta de vitalidad" de los centros de estudiantes. Mark van Aken, Los militantes. Historia del movimiento estudiantil uruguayo (Montevideo: FCU, 1990), 47-48. 
estudiantiles. Por otra parte, se fundó el Centro de Estudiantes Ariel, que congregó a quienes tenían "una gran inquietud intelectual” y estaban interesados por los "problemas sociales y educativos del país", pero no representaba a ninguna Facultad ni participaba como "entidad coordinadora central" de los universitarios ${ }^{22}$. El programa, publicado en Ariel, revista oficial del Centro de Estudiantes, reivindicaba el idealismo, la participación de la juventud y la prédica latinoamericana de José Enrique Rodó realizada en su ensayo Ariel $^{23}$ :
"Nosotros, levantamos ahora la bandera de Ariel: somos idealistas, confiamos en el poder de la voluntad, pedimos acción, nos mueve el optimismo y defendemos un concepto de patria que, sin perder el color local, pueda fundirse en el amplio concepto de América.
Tal nuestro programa; venimos a la lucha, confiados en la juventud y pugnaremos para que esa juventud moldee su espíritu al amparo de Ariel, de Ariel "genio del aire, imperio de la razón y el sentimiento sobre los bajos estímulos de la irracionalidad.
[...] Nuestro país, y al decir nuestro país decimos América, necesita acción: acción en los claustros y en el taller, acción en el silencio del estudio y en la serenidad de los campos, acción siempre"24.

La Comisión Directiva del Centro de Estudiantes Ariel efectuó una encuesta entre diferentes representantes sociales, políticos y estudiantiles para establecer los problemas de la sociedad y la función social de la Universidad ${ }^{25}$. Entre las respuestas recibidas y publicadas en la revista Ariel destaca la del maestro y escritor Alberto Lasplaces, en ese

22 Mark van Aken, Los militantes, 55.

23 En Ariel la "idealidad" consiste en la superioridad de la razón y un programa de acción para el perfeccionamiento moral y social de Latinoamérica: "Ariel, genio del aire, representa, en el simbolismo de la obra de Shakespeare, la parte noble y alada del espíritu. Ariel es el imperio de la razón y el sentimiento sobre los bajos estímulos de la irracionalidad; es el entusiasmo generoso, el móvil alto y desinteresado en la acción, la espiritualidad de la cultura, la vivacidad y la gracia de la inteligencia, el término ideal á que asciende la selección humana, rectificando en el hombre superior los tenaces vestigios de Calibán, símbolo de sensualidad y de torpeza, con el cincel perseverante de la vida". José Enrique Rodó, Ariel (Montevideo: Colombino Hermanos Limitada Editores, 1947), 47. "Ariel triunfante, significa idealidad y orden en la vida, noble inspiración en el pensamiento, desinterés en moral, buen gusto en arte, heroísmo en la acción, delicadeza en las costumbres". José Enrique Rodó, Ariel, 155.

24 "Nuestro programa", Ariel (julio 1919): 4.

25 "Nuestra encuesta", Ariel (septiembre 1919): 109. 
entonces Subdirector del Instituto Normal de Varones, donde consideraba la distribución de la tierra el mayor obstáculo para el progreso: "La democracia será una mentira entre nosotros mientras el país entero esté en manos de una pequeña minoría opulenta, en detrimento de una inmensa mayoría totalmente desposeída" ${ }^{26}$. Describía así la situación del campo uruguayo:

“El país está despoblado, y no se poblará mientras no sea posible la vida del hombre sobre la tierra. El proletariado rural que agrupa las tres cuartas partes de la población de la campaña constituye una rémora a todo adelanto, porque su falta de iniciativa, su pereza y su ignorancia, no son sino frutos lógicos de su miserable situación económica. El 50 por ciento, o más, de ese proletariado es analfabeto, y las lacras físicas, sobretodo la tuberculosis y la sífilis, hace en él estragos espantosos. ¿Qué civilización se pretende levantar sobre esa base? La nación no es estimable porque sus novillos pesen muchos kilos, ni porque sus ovejas den el vellón muy fino. La nación vale por sus hombres, y cuanto más libres, cultos y fuertes sean, más libre y culta y fuerte será ella misma"27.

Emilio Frugoni, abogado y Secretario General del Partido Socialista, coincidía en el diagnóstico: "Hay una campaña inculta y despoblada donde un proletariado nómade arrastra una lamentable vida de paria explotado y sumiso; hay desigualdades económicas irritantes; hay quienes monopolizan el territorio nacional; quienes monopolizan la fortuna; y hay quienes no pueden soportar la carestía de la vida o viven como las bestias, y hasta peor que las bestias". Además, destacaba la importancia de la Universidad como centro de "elaboración espiritual" donde se prepararían personas en contacto con la realidad social, para poder enfrentar "los problemas históricos, económicos y vitales del momento y desplegando una acción práctica de constante e inmediata utilidad colectiva, de acuerdo con el concepto de que la cultura debe ser un bien, una riqueza de las sociedades y para las sociedades, de donde en definitiva emana, y no un privilegio de los individuos para uso exclusivo de quienes lo atesoran" 28 .

26 Alberto Lasplaces, "Contestaciones a nuestra encuesta", Ariel (octubre-noviembre 1919): 178.

27 Alberto Lasplaces, "Contestaciones a nuestra encuesta", 178.

28 "Nuestra encuesta", Ariel (diciembre 1919-enero 1920): 221-222. 
En el mismo número, Ildefonso Pereda Valdez, vocal de la Comisión Directiva y redactor de la Comisión Revista, proponía replicar la experiencia de la Universidad de Oviedo (España) para poder "extender la cultura universitaria, filosófica, literaria o científica, hasta la masa obrera". Concebía la extensión universitaria como parte de la función social de la Universidad y establecía la responsabilidad del Centro de Estudiantes Ariel, como "institución cultural universitaria", de "velar por todo aquello que signifique extensión de esa misma cultura". Entendía que, de las cuatro formas de extensión utilizadas por la Universidad de Oviedo ${ }^{29}$, la más adecuada para realizar sería la "conferencia" o "conversación" a efectuarse en locales sindicales donde estudiantes expondrían sobre: Historia de la civilización, Sociología, Historia Natural, Derecho, Física, Química, Literatura, Lógica, Moral, Música, Arte, Economía y Geografía ${ }^{30}$. La Comisión Directiva atendió la propuesta y el 10 de junio de 1920 informaba a Emilio González, Secretario de la Federación Obrera Regional Uruguaya (FORU), el Plan de Extensión del Centro Ariel: "Organizar una serie de "conversaciones familiares' en los diversos locales obreros de la capital, que versarán sobre asuntos de ciencia, letras, artes, cuestiones de actualidad, etc., que estarán a cargo de estudiantes de distintas facultades"31.

El ciclo de conferencias se realizó los días “jueves y sábados por la noche", en el local central de la FORU (Río Negro, 1180), organizadas en dos categorías: por un lado, charlas de "cultura general" (disertaciones sobre José Enrique Rodó, León Tolstoi, Rafael Barret y Anatole France), y por otro cuestiones de higiene, prevención y alcoholismo (Primeros Auxilios, Higiene, Alcoholismo, Higiene de la boca, La sífilis y sus consecuencias y Problemas sexuales) ${ }^{32}$.

29 Las cuatro formas de extensión serían: "Conferencias en la Universidad”, "Conferencias pedagógicas para los maestros", "Clases especialmente destinadas a los obreros, y que son como el germen de la llamada "Universidad Popular" y "Lecciones fuera de la Universidad". La clasificación fue extraída del artículo de Francisco Giner de los Ríos, "La Universidad de Oviedo", publicado en el Boletín de la Institución Libre de Enseñanza y recopilado en la obra del mismo autor, La Universidad española (Madrid: Imp. Clásica Española, 1916), 294-297.

30 "Extensión universitaria", Ariel (diciembre 1919-enero 1920): 258-260.

31 "Extensión universitaria", Ariel (noviembre-diciembre 1920): 18

32 Las conferencias realizadas en la sede de la Federación Obrera Regional Uruguaya (FORU) fueron las siguientes:

- Bachiller Víctor Armand Ugón: 3 conferencias sobre el tema "Primeros Auxilios". 
La actividad fue catalogada como "éxito significativo", aún a pesar de las "dificultades existentes los primeros momentos" 33 . En agosto de 1920, a través del extenso editorial "Nuestro Programa" publicado en la revista Ariel, se ratificaba la obligación de organizar la Extensión Universitaria a través del Centro de Estudiantes Ariel o de la propia Universidad, como forma de "llevar al pueblo los conocimientos adquiridos en el aula" y se añadía la necesidad de establecer Universidades Populares como centros de cultura, "para ir, como sostenía Deherme, el apóstol de 'La cooperación de ideas', a la emancipación integral del proletariado" 34 .

\section{La Universidad Popular del Centro Ariel}

Finalmente, en 1930, la propuesta de creación de una Universidad Popular fue planteada en el Congreso Nacional de Estudiantes de la recientemente constituida Federación de Estudiantes Universitarios del Uruguay (FEUU), por el bachiller Héctor González Areosa, en representación del Centro de Estudiantes Ariel. El informe que acompañaba la iniciativa denunciaba las condiciones de vida de los trabajadores, la concentración de la propiedad de la tierra en manos de los grandes latifundistas ${ }^{35}$ y establecía la necesidad de crear Universidades Populares

- Bachiller Walberto Pérez: 3 conferencias sobre "Higiene".

- Doctor Joaquín de Salteráin: 2 conferencias sobre "Alcoholismo".

- Bachiller Carlos Quijano: 4 conferencias literarias:

- "La vida y la obra de Rodó".

- "Lectura comentada de Tolstoy".

- "Lectura comentada de Rafael Barret".

- "Lectura comentada de Anatole France".

- Dentista señor Roberto Quintana Moyano: 2 conferencias sobre "Higiene de la boca".

- Bachiller Aquiles Ibargoyen: 2 conferencias sobre "La sífilis y sus consecuencias", con proyecciones luminosas.

- Doctor Juan A. Collazo: 1 conferencia sobre "La prostitución".

También estaba proyectado realizar en la Casa del Pueblo, en el Centro "Brazo y Cerebro", y en el Ateneo de Montevideo: "2 conferencias sobre 'Higiene de la boca', a cargo del dentista A. Copello; 2 sobre 'Problemas sexuales', a cargo del bachiller Juan F. Canessa, y un curso de varias conferencias sobre 'Mecánica', a cargo del bachiller Luis Giorgi". "Extensión universitaria", Ariel (noviembre diciembre 1920): 19.

33 Siete años después, el propio Centro Ariel criticaba duramente las "fugaces" experiencias de extensión universitaria que no proporcionaban ningún resultado, ya que obedecían "más a un snobismo de muchachada ansiosa de lucirse que a una vocación sinceramente sentida". "El Centro de Chauffers y el proyecto de Universidad Popular", Ariel (diciembre 1927): 20.

34 "Nuestro programa", Ariel (agosto 1920): 5.

35 Poco tiempo después, lo mismo hacían los maestros: "El $50 \%$ de los propietarios poseía el $5 \%$ de 
con el apoyo de estudiantes, profesionales, intelectuales y dirigentes sindicales; con el objetivo de educar a las clases trabajadoras urbanas y rurales, y así hacerlas conscientes de su condición de explotadas.

\section{Imagen No. 1.}

Sesión inaugural del Primer Congreso Nacional de Estudiantes

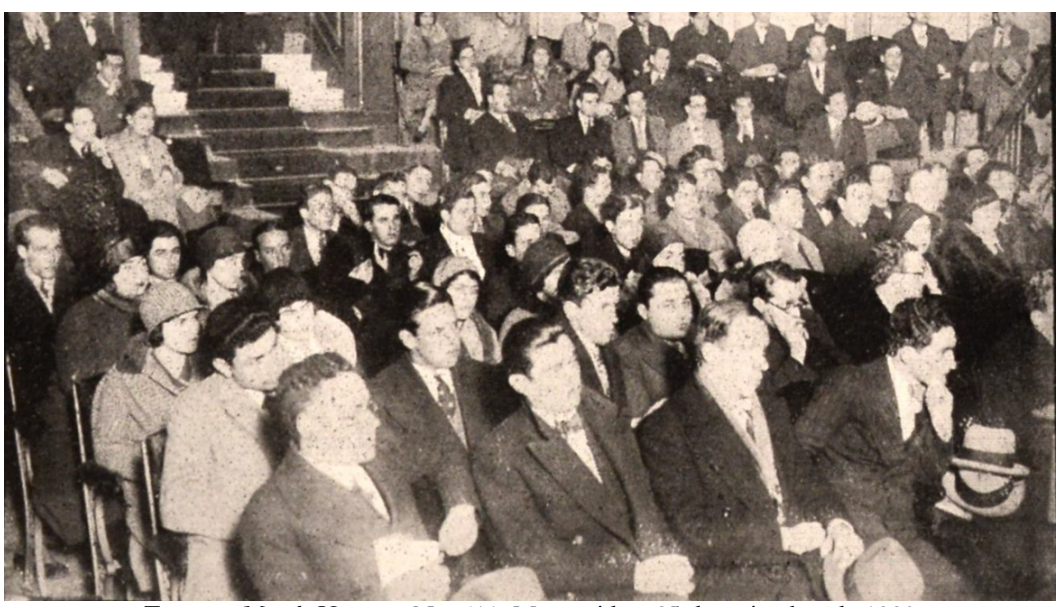

Fuente: Mundo Uruguayo No. 611, Montevideo, 25 de setiembre de 1930

Aprobada la moción, la Universidad Popular del Centro Ariel comenzó a funcionar el 14 de julio de 1931, en el recinto de la Escuela Superior de Comercio (ubicada en la calle Tristán Narvaja, hoy Emilio Frugoni, entre 18 de Julio y Guayabo) ${ }^{36}$, finalizando los cursos el 10 de noviembre ${ }^{37}$. La Secretaría, ubicada en la calle 18 de Julio, 1764, funcionaba entre las 18 y las 20 horas $^{38}$. En cambio, las clases se realizaban en horario nocturno desde las 20:05 hasta las 23:15, de lunes a viernes, aunque también los

la propiedad territorial rural; el $5 \%$ poseía el $50 \%$ de la tierra y el restante $45 \%$ poseía el $45 \%$ de la propiedad rural. [...] La presencia de los rancheríos, forma extrema del minifundio. [...]. Julio Castro estimaba su número en 587, con aprox. 120.000 habitantes, entre ellos de 20.000 a 25.000 niños en edad escolar, personas, dice Castro, 'que viven por debajo de lo que podrá considerarse nivel de vida humano". Miguel Soler, "El movimiento en favor de una nueva escuela rural", en Dos décadas en la historia de la escuela uruguaya. El testimonio de los protagonistas, ed. AA.VV. (Montevideo: Ediciones de la Revista de la Educación del Pueblo, 1987), 63.

36 El historiador Mark Van Aken señala erróneamente que la Universidad Popular comenzó a funcionar en "salones ofrecidos por la Facultad de Derecho". Mark van Aken, Los militantes, 73.

37 "HOY CLAUSURA SUS CURSOS LA UNIVERSIDAD POPULAR - Hoy martes, a la hora 21, en el local de la Escuela S. de Comercio, calle Tristán Narvaja y 18 de Julio, se efectuará el acto de clausura de los cursos de la Universidad Popular. Con tal motivo, se exhorta a los alumnos y profesores para que concurran a dicho acto y se invita para participar en el mismo a las personas e instituciones que simpaticen con la obra de la Universidad Popular. LA SECRETARIA". "Universidad Popular", El Sol (10 de noviembre de 1931): 2.

38 "Universidad Popular", El Sol, (miércoles 27 de abril de 1932). 
sábados se realizaban actividades ${ }^{39}$. Los cursos, gratuitos y de asistencia libre, se encontraban divididos en regulares y especiales. Mientras los regulares duraban todo el año, los especiales eran breves y puntuales sobre determinados temas.

Al segundo año de funcionamiento los cursos regulares eran: Economía Política, Literatura, Filosofía, Historia Universal, Historia del Arte, Historia Natural, Dibujo, Aritmética, Gramática, Contabilidad, Taquigrafía, Higiene y Profilaxis, Francés, Inglés, Alemán, Esperanto, Física, Electricidad, cursos de primaria para analfabetos y Lecturas comentadas.

Los especiales, eran brindados por reconocidos intelectuales de izquierda y de los sectores progresistas de los partidos tradicionales. Las temáticas fueron las siguientes:

- Legislación Obrera Nacional Aplicada: Emilio Frugoni ${ }^{40}$.

- Marxismo: P. Ceruti Crosa ${ }^{41}$.

- El esfuerzo del hombre en la conquista de la Verdad: Clemente Estable $^{42}$.

- Las Reformas Agrarias: Carlos Quijano ${ }^{43}$.

39 "Hoy sábado 8, a las 21 horas, en el local de la Universidad Popular, calle Tristán Narvaja casi esquina 18 de julio, el estudiante Alfredo Aragona sobre 'La Nueva Arquitectura y su Función Social'. La disertación será ilustrada con proyecciones luminosas". "Universidad Popular", La Tribuna Popular (6 de agosto de 1931): 3.

40 Emilio Frugoni (1880-1969), abogado, escritor, poeta, docente liceal y universitario, decano de la Facultad de Derecho, y diputado socialista uruguayo. Véase: Carlos Real de Azúa, Antología del ensayo uruguayo contemporáneo, Vol. 1 (Montevideo: Universidad de la República, 1964), 111-129.

41 Pedro Ceruti Crosa (1899-1947), abogado y diputado del Partido Colorado. En 1925 se incorpora a la sección uruguaya del Socorro Rojo Internacional y en la década del treinta termina afiliándose al Partido Comunista del Uruguay. Eugenio Gómez, Historia del Partido Comunista del Uruguay hasta el año 1951 (Montevideo: Editorial Eco, 1990), 69; Gerardo Leibner, Camaradas y compañeros. Una historia política y social de los comunistas del Uruguay. Tomo I: La era Gómez, 1941-1955 (Montevideo: Trilce, 2011), 287.

42 Clemente Estable (1894-1976), maestro y biólogo, Maestro de Conferencias, Adjunto a la Inspección Técnica de Enseñanza Primaria. En 1922 obtuvo una beca ofrecida por el gobierno español para estudiar junto al premio Nobel Santiago Ramón y Cajal. De regreso a Uruguay se integró como Jefe de Sección al naciente Instituto de Neurología y en 1927 se le creó, dentro del Consejo de Enseñanza Primaria y Normal, el Laboratorio de Ciencias Biológicas. Ángela de Sosa y Roselén Schiaffino, "Clemente Estable... En una encrucijada ¿Pedagogo, científico o filósofo?”, Ixtli. Revista Latinoamericana de Filosofía de la Educación Vol. 4, No. 7 (2017): 99-116.

43 Carlos Quijano (1900-1984), abogado, político, ensayista y periodista uruguayo. Fundador en 1917, junto con otros jóvenes reformistas, del Centro de Estudiantes Ariel. A los 23 años recibió el título de abogado y partió a estudiar Economía y Ciencias Políticas en la Universidad de la Sorbona de 
- El derecho, la justicia y las luchas sociales: Lorenzo Carnelli44.

- Historia del movimiento obrero: F. Carreño.

- La Novela Americana: Enrique Centrón ${ }^{45}$.

- El Imperialismo: Arturo Prunell.

\section{El surgimiento de las Universidades Populares en los BARRIOS}

El 1 de marzo de 1931 asumió la Presidencia de la República el Dr. Gabriel Terra. Dos años después dio un Golpe de Estado, disolvió el Parlamento y, para legitimar sus acciones, convocó a una Convención Constituyente que elaboró una nueva Constitución, la cual fue plebiscitada y aprobada en 1934. Junto con el plebiscito de la Constitución se eligió al presidente Gabriel Terra, por segunda vez. La reforma constitucional le dio mayor poder de decisión, que utilizó para favorecer a los estancieros, los bancos y las empresas extranjeras, así como para detener las reformas que había impulsado el batllismo.

La represión y la destitución fueron recursos utilizados por las autoridades educativas para disciplinar a los docentes. La resistencia a través de huelgas y movilizaciones de los maestros tuvieron como respuesta traslados, destituciones y suspensiones en todo el país: Selmar Balbi va a ser destituido por enseñar en la Universidad Popular de Dolores ${ }^{46}$; Julio Castro, suspendido quince días "en oportunidad de haber pronunciado una conferencia en el Museo Pedagógico”, donde se había expresado en "términos irrespetuosos" mientras realizaba un análisis "tendencioso

París. Participó en la Asociación General de Estudiantes Latinoamericanos (AGELA) junto a Víctor Raúl Haya de la Torre, Julio Antonio Mella, Carlos Pellicer, Miguel Ángel Asturias, Toño Salazar y León de Bayle. En 1928 retornó y fundó la Agrupación Nacionalista Demócrata Nacional dentro del Partido Nacional, con la que accedió a una banca de diputado. Fue uno de los fundadores del Frente Amplio en 1971. En 1939 fundó y dirigió el semanario Marcha, hasta su clausura el 22 de noviembre de 1974 por parte de la dictadura cívico-militar. Se exilió en México en noviembre de 1975. Gerardo Caetano y José Pedro Rilla (1986), El joven Quijano (1900-1933).

44 Lorenzo Carnelli (1887-1960), abogado y político uruguayo. Fundador del Partido Blanco Radical, escindido del Partido Nacional, "identificado con el reformismo económico-social”. B. Nahum, A. Cocchi, A. Frega y Y. Trochon, Crisis política y recuperación económica, 1930-1958. Historia Uruguaya, Tomo 9 (Montevideo: Ediciones de la Banda Oriental, 2011), 15.

45 Enrique Centrón, abogado y profesor de literatura. Arturo Ardao, "Viejos recuerdos del Centro de Estudiantes de Derecho", Revista del Centro de Estudiantes de Derecho 92 (1967): 14.

46 "Una enormidad", El País (3 de junio de 1936): 5. 
de la religión católica y el fascismo" ${ }^{47}$. En los Institutos Normales de Montevideo se desató una "verdadera oleada represora" ${ }^{48}$, no solamente contra los docentes:

"Los alumnos normalistas, convertidos en agentes activos del comunismo, provocando desórdenes, obstruyendo la obra de la Enseñanza, alterando la disciplina de la Escuela y agitando la opinión contra el Estado, no solo ejercen una actividad que es dudosamente lícita, sino que no deben recibir un diploma que los habilite legalmente para ejercer el Magisterio Nacional, al servicio del Estado y subordinados al Estado que niegan, y acusan y desprestigian"49.

La proliferación de las Universidades Populares comenzó a partir del año 1936, "cuando se corrió la voz de que las nacientes instituciones iban a trabajar para debilitar el régimen de Terra" 50 . Las Universidades Populares se encontraban principalmente en distintos barrios de Montevideo: Villa Muñoz; Villa Dolores (Universidad Popular "Florencio Sanchez"); la de la Unión (Universidad Popular "José E. Rodó”), Barrio Sur, Cerro, Barrio Olímpico, Universidad Popular "Clemente Estable".

En un intento de ordenar el funcionamiento de las Universidades Populares comenzó a trabajar un Consejo Coordinador, del que formaban parte los delegados de las diferentes instituciones y Agustín Ruano Fournier, conjuntamente con Ulises Riestra y Clemente Estable en carácter de delegados del Ateneo de Montevideo. La gestión del Consejo Consultivo tenía tres objetivos: a) Organizar las Universidades Populares; b) Ordenar el sistema de enseñanza de acuerdo con el medio en el que se encontraba; c) Cultura artística popular ${ }^{51}$.

47 Foja de servicio del maestro Julio Castro, disponible en el Archivo Histórico de los Institutos Normales de Montevideo.

48 Mónica Maronna, "La dictadura y la enseñanza secundaria", en El Uruguay de los años treinta: enfoques y problemas, eds. Mónica Oribe Cures et al. (Montevideo: Ediciones de la Banda Oriental, 1994): 181.

49 José Claudio Williman, Memoria de Enseñanza Primaria y Normal, correspondiente a los años 1934 y 1935, presentada al Consejo Nacional de Enseñanza Primaria y Normal por su Presidente José Claudio Williman (Montevideo: s/e, 1937), 94.

$50 \quad$ Mark van Aken, Los militantes, 75.

51 “El Dr. A. Ruano Fournier nos habla de las Universidades Populares", El Plata (23 de julio de 1937):3. 
En la mayoría de las instituciones funcionaban cursos de Inglés, Francés, Castellano, Alemán, Contabilidad, Aritmética, Caligrafía, Ortografía, Taquigrafía, Gramática, Corte y Confección, Labores y Electricidad. Incluso se realizaban, en pocos centros y con irregularidad, cátedras de Literatura, Filosofía, Historia y Economía Política ${ }^{52}$.

El profesorado de las Universidades Populares se constituía de estudiantes, empleados, maestros y profesionales universitarios:

"Estudiantes que roban tiempo a su propio aprendizaje para enseñar a quienes van a adquirir conocimientos elementales; maestros que luego de cuatro horas de clase en las escuelas públicas, concurren a inculcar sus enseñanzas a otros elementos anhelosos de asimilarlas; catedráticos de Facultades y Liceos que conocen a fondo su misión adoctrinadora y la necesidad que de ella tiene un gran sector social; contadores públicos, tenedores de libros, empleados de Banco y de Comercio que desechan el merecido reposo después de la labor diaria para dictar, dos o tres veces por semana, su clase de conocimientos a quienes desean aprender o especializarse en determinados aspectos del saber humano"53.

Los periódicos partidarios del gobierno, por el contrario, afirmaban que las Universidades Populares eran "templos levantados a la devoción de Lenin" 54 , donde algunos profesores enseñaban el "internacionalismo-extremista", otros glorificaban el "homicidio filosófico-político" y los más estaban "desvinculados del ennoblecedor sentimiento de nacionalidad" 55 . Para impedirlo, el Poder Ejecutivo presentó una ley de fiscalización de las instituciones privadas, con la cual se evitaría la alteración de "sus preocupaciones puramente docentes, hacía el campo de la lucha social, como abanderados de fracasadas y brutales teorías sovietizantes", pero fue rechazada en el Parlamento ${ }^{56}$.

52 "Los cursos que dictan las Universidades Populares. Se da preferencia a los de especialización mercantil o aprendizaje manual", El Plata (14 de julio de 1937): 3.

53 "El profesorado de las Universidades Populares constituye una prueba más de la solidaridad social", El Plata (12 de julio de 1937): 3.

54 "Mala Escuela", El Pueblo (22 de noviembre de 1936): 16.

55 “Las 'Inofensivas' Universidades 'Populares”, El Pueblo (15 de julio de 1937): 16.

56 "Profilaxia Urgente", El Pueblo (12 de julio de 1937): 16. 


\section{ALTOS ESTUDIOS}

\section{Imagen No. 2}

- ¿Cuál es el país más feliz de la tierra? - Rusia. - ¡Bien!... Dame un ejemplo de algún otro país que ya esté en el camino de esa felicidad. - España... ¡Muy bien! Y ahora, una última pregunta: ¿Cuál es la escuela mejor para preparar a la juventud para la futura felicidad? - ¡La Universidad Popular! - ¡Estupendo!

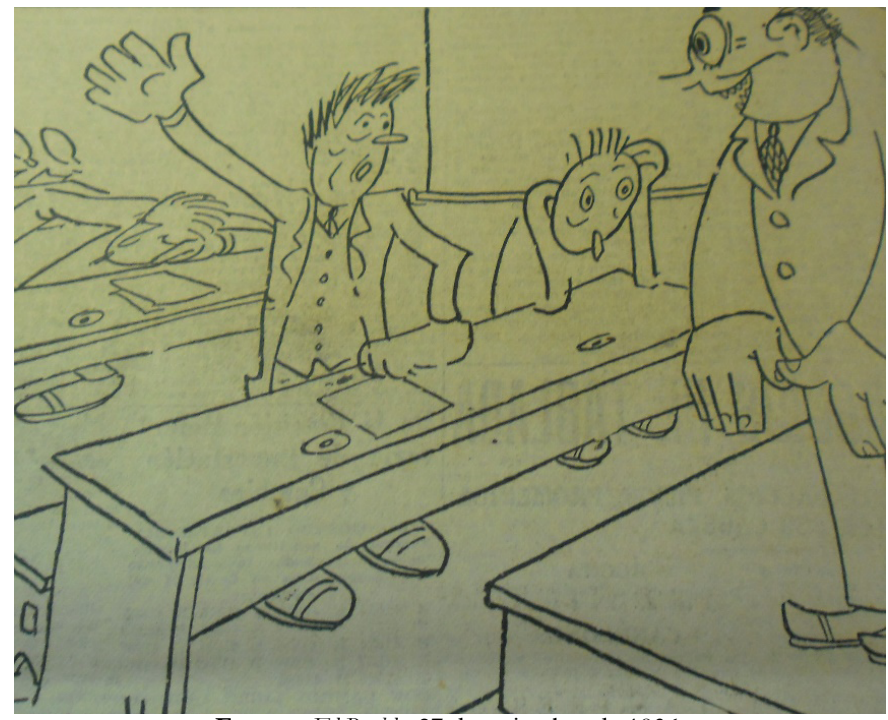

Fuente: El Pueblo, 27 de setiembre de 1936.

\section{Imagen No. 3}

\section{SE EXPLICA}

- ¿Por qué llora tu nene? - Porque en la Universidad Popular de la esquina, se le quedaron con el balero y le dieron un martillo. Figúrate, ahora quiere clavos...

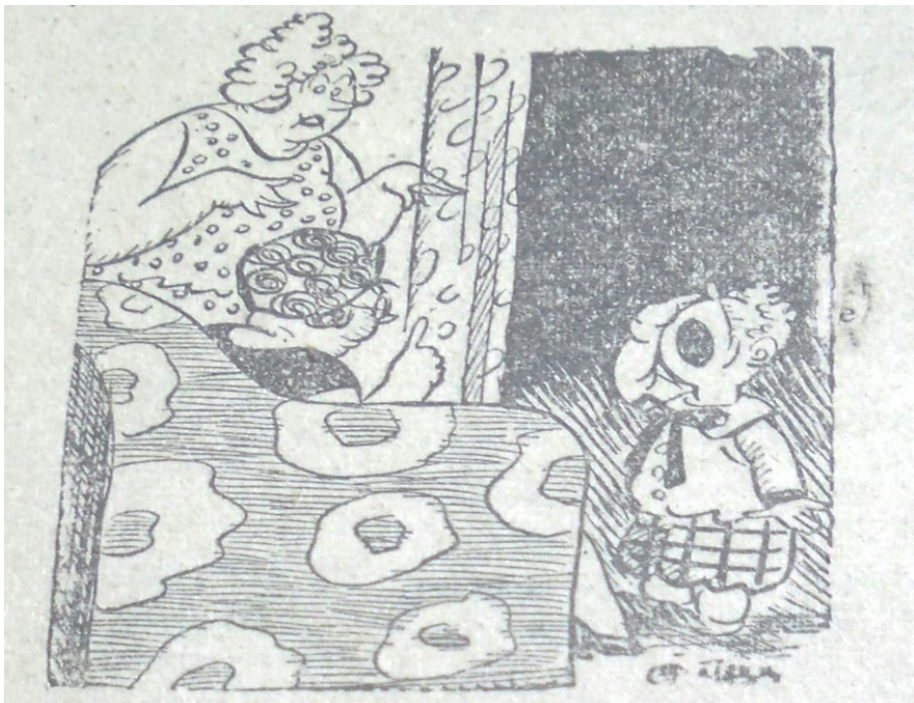

Fuente: El Pueblo, 18 de junio de 1937. 


\title{
5. La Universidad Popular Central
}

Entre todas las Universidades Populares, la más prestigiosa fue la Universidad Popular Central. Fue fundada el 27 de abril de $1938^{57}$, con representantes de diversas instituciones como el Ateneo de Montevideo, la Federación de Estudiantes Universitarios del Uruguay (FEUU) ${ }^{58}$, la Unión Nacional del Magisterio, la Agrupación de Intelectuales, Artistas, Periodistas y Escritores (AIAPE) y la Escuela Taller de Artes Plásticas (ETAP):

\begin{abstract}
"Ha surgido la iniciativa, ya en andamiento, de fundar una gran entidad con la denominación de UNIVERSIDAD POPULAR CENTRAL. Para la plena realización de este propósito, que concita nuestros mejores afanes, es preciso agrupar en torno de la nueva institución a los ciudadanos que en el momento histórico actual afirman con dignidad y resolución su fe en los auténticos principios democráticos que postulan una constante y libre actividad cultural en el seno del pueblo"59.
\end{abstract}

Finalizada la presidencia de Gabriel Terra, la "penetración fascista" en el sistema educativo ${ }^{60}$ y el "desastre de nuestra escuela primaria" eran la demostración "más categórica y valedera al por qué de su existir"61. Las Universidades Populares, "tantas veces calumniadas e injustamente

57 La resolución del Consejo Coordinador de las Universidades Populares del Uruguay, de enero de 1938, estableció la creación de la Universidad Popular Central "sobre la base" de la Universidad Popular del Barrio Olímpico. Gabriel del Mazo (comp.), La Reforma Universitaria, Tomo II, 405. El 27 de marzo de 1938 se produjeron las elecciones nacionales donde el Gral. Alfredo Baldomir se convirtió en Presidente de la República, finalizando el período terrista.

58 La Federación de Estudiantes Universitarios del Uruguay se fundó el 26 de abril de 1929. Estuvo integrada en sus inicios por la Asociación de Estudiantes de Medicina, Asociación Uruguaya de Estudiantes de Notariado, Asociación de Estudiantes de Agronomía, Centro de Estudiantes de Derecho, Asociación Estudiantes de Farmacia, Centro de Estudiantes de Arquitectura, Centro de Estudiantes de Odontología, Asociación de Estudiantes de Veterinaria, Centro de Estudiantes de Ingeniería y Centro Cultural Liceo Nocturno. Francisco Sanguiñedo, La FEUU ayer y hoy. 70 años de documentos del Movimiento Estudiantil Uruguayo (Montevideo: Tradinco, 2014).

59 "Se ha creado una gran Universidad Popular Central. Se instalará en 18 de Julio y Ejido", Acción (12 de marzo de 1938): 2.

60 Véanse "Conspirando contra la Independencia Nacional", Marcha (23 junio de 1939): 10; "El partido Nazi en el Uruguay es un foco de espionaje", Marcha (30 junio de 1939): 8; "Como se organiza la quinta columna de la traición”, Marcha (7 julio de 1939): 8. También: Hugo Fernández Artuccio, Nazis en el Uruguay (Montevideo: Talleres Gráficos del Sur, 1940).

61 "Vasta labor desarrolla la Universidad Popular Central", Boletín de la Universidad Popular Central (setiembre de 1939): 2. 
combatidas", tenían un "deber nacional", "supliendo las escuelas que faltan y complementando la acción insuficiente y precaria de las que existen"

En la Universidad Popular Central los cursos eran gratuitos y se confeccionaban según solicitudes, propuestas y sugerencias de los propios estudiantes. Comprendían desde alfabetización, pasando por los de capacitación técnica, hasta los cursos superiores o de ampliación cultural: Analfabetos y Semianalfabetos; Aritmética, Ortografía y Redacción; Gramática, Corte y Confección, Ciencias Geográficas, Francés, Inglés, Contabilidad, Taquigrafía, Dibujo lineal, Dibujo artístico, Castellano para extranjeros, Ingreso a Secundaria, Solfeo, Encuadernación, Economía Política, Anatomía, Fisiología e Higiene.

La creación de la Sección Interior en la Universidad Popular Central va a estar formada, fundamentalmente, por integrantes destacados de la Unión del Magisterio. El principal objetivo de la Sección Interior era la difusión de la cultura en toda la República, propiciando relaciones de intercambio y ayuda mutua con diversas instituciones culturales y deportivas como "Renovación", de Florida; "Cultura y de Deporte", de Melo; "José Enrique Rodó”, de Juan L. Lacaze; "Elevación”, de Dolores; "Escuela Experimental", de Progreso; "Gabriela Mistral", de Canelones, y "Florencio Sánchez", de Dolores ${ }^{63}$. Entre las actividades realizadas se destacaban:

- Donaciones de libros, destinadas a aumentar los fondos de las Universidades y Bibliotecas Populares del interior.

- Ciclos de Conferencias, organizadas junto a la Comisión de Cultura, a cargo de distintos periodistas y escritores. En el Primer Ciclo intervinieron los escritores Justino Zavala Muniz, Serafín J. García, Juan José Morosoli, Santiago Dossetti, Francisco Espínola y Alfredo Lepro. Todos coincidían en el diagnóstico: el latifundio, el minifundio, el analfabetismo, las enfermedades 
y vicios sociales, eran resultado de la falta de trabajo y de la pobreza en el medio rural. Asimismo, reclamaban la creación de Universidades Populares "apartadas de los rigorismos oficiales, de las retóricas, de las necesidades utilitarias y, naciendo en el seno del pueblo, del pueblo y para el pueblo" ${ }^{64}$.

En 1941, Danilo Trelles realizaba una evaluación del trabajo realizado por las Universidades Populares en el campo:

"Allí no hay medios con qué lograr una cultura. Que vaya alguno a convencerlos de que es necesario instruirse, y se le reirán en la cara. Y tendrán toda la razón del mundo. Cuando urgencias económicas torturan el estómago, no hay tiempo para pensar en eso. Podría hacerlo el hijo del estanciero o del chacrero rico, cuyo esfuerzo no fecunda la simiente, cuyos dedos no desmenuzan los terrones, y que tiene el espíritu tan lejos de todo esto"65.

Testimonio coincidente con la valoración que realizará años después el maestro Julio Castro, refiriéndose a experiencias anteriores durante la primera Misión Socio-Pedagógica al rancherío ${ }^{66}$ de Caraguatá: "Con cultura sólo en campaña, no hacemos nada" ${ }^{67}$.

\section{Conclusiones}

La década del 30 está atravesada en Uruguay por el primer golpe de Estado que se produce en el siglo XX, que fue dado por el presidente

64 J. Zavala Muniz, "Virtudes criollas", Marcha (27 de setiembre de 1940): 18.

65 Danilo Trelles, "Razón y destino de las Universidades Populares", Marcha (31 de enero de 1941): 12.

66 "[Un] Conjunto de viviendas de la gente que ya no tiene lugar determinado en la vida social y económica y se ha ido a establecer en esos lugares. No sabemos aún qué factores determinan la selección del lugar para este agrupamiento humano. Quizás la equidistancia de sus primitivos sitios de residencia. La existencia de leña y agua, la disponibilidad de tierras fiscales o "tierras de nadie", o la proximidad a la estancia o al caserío, que puedan ofrecer oportunidad de trabajo para hombres y mujeres. El hecho es que en la vasta campiña uruguaya, agrícola o ganadera existe el rancherío. [...] Albergan a más de cien mil personas, las que están viviendo en las más terribles condiciones humanas, las que han llevado a desmejorar con el agraviante nombre de 'pueblos ratas"'. Víctor Emilio Silveira, Las Misiones Sociopedagógicas en el Uruguay (Washington D. C.: Unión Panamericana, Publicaciones del Centro Interamericano de Educación Rural, 1960), 5-6.

67 Julio Castro, "La misión pedagógica de los alumnos normalistas", Cuadernos de Marcha, Tercera Época, Año I, No. 7 (1985): 22. 
electo para impulsar una reforma constitucional. En un contexto signado por una crisis económica, los sectores conservadores tomaron la iniciativa intentando realizar una serie de reformas. En la enseñanza ocurrió algo similar, intentando implantar un orden autoritario a través de la reforma de las instituciones educativas o la represión y destitución de los docentes. Entonces, las Universidades Populares se constituyeron en un refugio para docentes, intelectuales y trabajadores contra el régimen terrista, siendo el período de su mayor expansión. El proyecto de ley presentado por parte del gobierno de Gabriel Terra para controlar o destruir a las Universidades Populares es una clara demostración de la preocupación que generaban en los sectores conservadores y de derechas.

Acabado el período terrista y reincorporados los estudiantes y profesores destituidos a las diferentes instituciones educativas oficiales, la Universidad Popular Central logró mantenerse hasta el año $1942^{68}$. El cierre implicó la terminación del intento de democratizar el acceso a la cultura en el medio rural. Sin embargo, el diagnóstico y análisis de la situación fue determinante para que, tres años después, surgieran las Misiones Socio-Pedagógicas, organizadas por estudiantes de Magisterio a los que se irían sumando los de Medicina, de Odontología, de Agronomía, de la Universidad del Trabajo. Llevaron cine, títeres, teatro, juegos, baile y música a la escuela rural y procuraron acercar prácticas de higiene, de salud y de vivienda allí donde la pobreza daba cuenta del abandono en que transcurría la vida de la inmensa mayoría de la población rural ${ }^{69}$.

Por último, las Universidades Populares en Uruguay surgen a iniciativa de los estudiantes universitarios, pero es con la incorporación de los trabajadores cuando alcanzan la mayor expansión y desarrollo. También la convergencia del movimiento obrero y el estudiantil lograda en las Universidades Populares confluirá en el apoyo a la aprobación de la Ley Orgánica de 1958, donde se establece la autonomía y el co-gobierno para la Universidad de la República.

69 Véase María García Alonso y Gabriel Scagliola (eds.), Misiones Socio-pedagógicas de Uruguay (1945-1971). Documentos para la memoria (Montevideo: Administración Nacional de Educación Pública-ANEP, 2012). 


\section{BibLIOgRAFÍA}

\section{Fuentes primarias}

\section{Archivo}

Archivo Histórico de los Institutos Normales de Montevideo, Montevideo - Uruguay.

\section{Diarios}

Acción.

El País.

El Plata.

El Pueblo.

La Tribuna Popular.

\section{Semanarios}

El Sol.

Marcha.

Mundo Uruguayo.

\section{Revistas}

Anales de la Liga de Estudiantes Americanos.

ALAPE Por la Defensa de la Cultura. Órgano de la Agrupación de Intelectuales, Artistas, Periodistas y Escritores (Sección Uruguaya).

Ariel. Revista del Centro de Estudiantes "Ariel".

Cuadernos de Marcha

El Estudiante Libre.

Evolución. Órgano de la Federación de los Estudiantes del Uruguay.

\section{Boletines}

Boletín de la Universidad Popular Central.

\section{Documentos}

Diario de Sesiones de la Honorable Cámara de Representantes. $47^{\mathrm{a}} \mathrm{Se}$ sión Extraordinaria, 25/11/1911. Sesiones extraordinarias del primer período de la XXIV Legislatura, Tomo CCXIII, octubre 19 a diciembre 9 de 1911. Montevideo: El Siglo Ilustrado, 1912. 
Williman, José Claudio. Memoria de la Enseñanza Primaria y Normal, correspondiente a los años 1934 y 1935, presentada al Consejo Nacional de Enseñanza Primaria y Normal por su Presidente José Claudio Williman. Montevideo: s/e, 1937.

\section{Libros}

Giner de los Ríos, Francisco. "La Universidad de Oviedo". En la obra del mismo autor, La Universidad española. Madrid: Imp. Clásica Española, 1916, 273-301.

Palacios Morini, Leopoldo. Las Universidades Populares. Valencia: F. Sempere y Compañía Editores, 1908.

Rodó, José Enrique. Ariel. Montevideo: Colombino Hermanos Limitada Editores, 1947.

\section{Fuentes secundarias}

Ardao, Arturo. La Universidad de Montevideo. Su evolución histórica. Montevideo: Ediciones Universitarias, 2008.

Ardao, Arturo. "Viejos recuerdos del Centro de Estudiantes de Derecho”, Revista del Centro de Estudiantes de Derecho 92 (1967): 4.

Bralich, Jorge. La Extensión Universitaria en el Uruguay. Montevideo: Comisión Sectorial de Extensión y Actividades en el Medio, 2007.

Caetano, Gerado y José Pedro Rilla. El joven Quijano, 1900-1933: izquierda nacional y conciencia crítica. Montevideo: Ediciones de la Banda Oriental, 1986.

Del Mazo, Gabriel (comp.). La Reforma Universitaria. La Plata: Centro de Estudiantes de Ingeniería, 1941, 3 tomos.

Delio Machado, Luis $\mathrm{M}^{\mathrm{a}}$ y Alfredo Alpini. "La educación secundaria y superior entre 1903 y 1935”. En Historia de la educación uruguaya. Tomo 3: La educación uruguaya 1886-1930, ed. Agapo Palomeque (Montevideo: Ediciones de la Plaza, 2012), 291 - 332.

Fernández Artuccio, Hugo. Nazis en el Uruguay. Montevideo: Talleres Gráficos del Sur, 1940.

García Alonso, María y Gabriel Scagliola (eds.). Misiones Socio-pedagógicas de Uruguay (1945-1971). Documentos para la memoria. Montevideo: Administración Nacional de Educación Pública-ANEP, 2012. 
Gómez, Eugenio. Historia del Partido Comunista del Uruguay hasta el año 1951. Montevideo: Editorial Eco, 1990.

Leibner, Gerardo. Camaradas y compañeros. Una historia política y social de los comunistas del Uruguay. Tomo I: La era Gómez, 19411955. Montevideo: Trilce, 2011.

Markarián, Vania, María Eugenia Jung e Isabel Wschebor. 1908. El año augural. Montevideo: Universidad de la República, 2008.

Maronna, Mónica. "La dictadura y la enseñanza secundaria". En El Uruguay de los años treinta: enfoques y problemas, editado por Mónica Oribe Cures et al. Montevideo: Ediciones de la Banda Oriental, 1994.

Nahum, Benjamín (coord.). Estadísticas históricas del Uruguay, 19001950. Tomo I: Población y Sociedad, Política, Educación, Estado. Montevideo: Universidad de la República, 2007.

Nahum, Benjamín. La época batllista, 1905-1929, Tomo 8. Montevideo: Ediciones de la Banda Oriental, 2011.

Nahum, B., A. Cocchi, A. Frega y Y. Trochon. Crisis política y recuperación económica, 1930-1958. Historia Uruguaya, Tomo 9. Montevideo: Ediciones de la Banda Oriental, 2011.

Oddone, Juan y París de Oddone, Blanca. Historia de la Universidad de la República. Tomo II: La Universidad del militarismo a la crisis. Montevideo: Ediciones Universitarias, 2010.

Rama, Carlos. Batlle: la conciencia social. Enciclopedia Uruguaya, Vol. 34. Montevideo: Arca, 1968.

Real de Azúa, Carlos. Antología del ensayo uruguayo contemporáneo, Vol. 1. Montevideo: Universidad de la República, 1964.

Sanguiñedo, Francisco. La FEUU ayer y hoy. 70 años de documentos del Movimiento Estudiantil Uruguayo. Montevideo: Tradinco, 2014.

Silveira, Víctor Emilio. Las Misiones Sociopedagógicas en el Uruguay. Washington D. C.: Unión Panamericana, Publicaciones del Centro Interamericano de Educación Rural, 1960.

Soler, Miguel. "El movimiento en favor de una nueva escuela rural". En Dos décadas en la historia de la escuela uruguaya. El testimonio de los protagonistas, editado por AA.VV. Montevideo: Edición de la Revista de la Educación del Pueblo, 1987, 27-73. 
Sosa, Ángela y Roselén Schiaffino. "Clemente Estable... En una encrucijada ¿Pedagogo, científico o filósofo?”. Ixtli. Revista Latinoamericana de Filosofía de la Educación Vol. 4, No. 7 (2017): 99-116.

Van Aken, Mark. Los militantes. Historia del movimiento estudiantil uruguayo. Montevideo: FCU, 1990.

Yaffé, Jaime. Ideas, programa y política económica del batllismo. Uruguay 1911-1930. Montevideo: Instituto de Economía, Facultad de Ciencias Económicas y de Administración, 2000.

Para citar este artículo: Scagliola, Gabriel. "Las Universidades Populares en Uruguay (1930-1942)", Historia Caribe Vol. XVI No. 38 (Enero-Junio 2021): 81-106. DOI: https://doi.org/10.15648/ hc.38.2021.2812 